\title{
On the relevance of Born theory in global seismic tomography
}

Lapo Boschi

ETH Zürich, Switzerland.

Thorsten W. Becker

University of Southern California, USA.

Gaia Soldati

INGV Roma, Italy.

Adam M. Dziewonski

Harvard University, USA.

L. Boschi, Institute of Geophysics, ETH Zürich, HPP O13.2 Hönggerberg, CH-8093 Zürich, Switzerland. (lapo@erdw.ethz.ch)

D R A F T

January 30, 2006, 3:13pm

D R A F T 
Does the application of seismic Born theory, as opposed to simpler ray theory, lead to an improvement in tomographic images of the Earth? In recent publications, Montelli et al. (2004a, 2004b) and van der Hilst \& de Hoop (2005) among others have expressed opposite opinions. We propose a quantitative approach to the comparison of tomographic images, which we apply to the case of surface-wave phase velocity maps derived with Born vs. ray theory. 


\section{Introduction}

Application of Born theory (sometimes referred to as finite-frequency theory, scattering theory, "banana-doughnut" effect...) in the context of global seismic tomography is not novel in itself (Woodhouse \& Girnius 1982; Snieder \& Nolet 1987; Li \& Tanimoto 1993; Li \& Romanowicz 1996), but has received much attention over the last few years (Dahlen et al. 2000; Hung et al. 2000; Zhao et al. 2000; Spetzler et al. 2001, 2002; Sieminski et al. 2004; Yoshizawa \& Kennett 2005; Zhou et al. 2005), as a fast progress in computational capabilities, accompanied by methodological improvements, has made it practical. Most recently, Montelli et al. (2004a, 2004b) have derived a new, global P-wave velocity model of the Earth's mantle in the framework of Born theory; they estimated their "finitefrequency" model to be characterized by velocity perturbations with "amplitudes 30-50 per cent larger than in the corresponding ray-theoretical images"; more importantly, they stated that their new images "provide clear evidence that a limited number of hotspots are fed by plumes originating in the lower mantle" (Montelli et al. 2004b). Montelli et al.'s (2004a, 2004b) claims have been questioned by many authors. The work of de Hoop \& van der Hilst (2005a), Dahlen \& Nolet's (2005) comment and the subsequent new articles by de Hoop \& van der Hilst (2005b) and van der Hilst \& de Hoop (2005) are examples of an animated debate. We wish to add a few remarks, with the intent of exploring in more depth some of the issues in discussion.

\section{P-velocity Maps of the Earth's Mantle from Ray and Born Theory}

Montelli et al. (2004a, 2004b) have evaluated the effect of applying Born theory to global P-wave travel-time tomography, carrying out both ray- and Born-theory inversions 
of the same database, and comparing the results. Albeit small, they claim differences between the ray- and Born-theory maps to be significant.

While confirming, to some extent, the large scale pattern of other models, the new Princeton models (both ray- and Born-theoretical) stand out as very radially coherent, with many slow and fast anomalies extending across the $660 \mathrm{~km}$ discontinuity. Vertical coherence is highest in the Born-theoretical images of Montelli et al. (2004a), with slow anomalies of relatively small lateral extent resembling vertical plumes under many hotspots. In view of such correlation between tomographic results and expected (or, at least, plausible) geophysical features, Montelli et al. (2004a) conclude that the growth in radial coherence represents an improvement in tomographic resolution.

We compute the radial correlation matrix $r\left(z_{1}, z_{2}\right)$ (Puster \& Jordan 1997; Becker \& Boschi 2002) between lateral structure at any two depths $z_{1}$ and $z_{2}$, and find that the Born-theoretical model by Montelli et al. (2004a) is characterized by a diagonal band of high radial correlation (figure 1a) much larger than that resulting, for example, from our independent inversion (Soldati \& Boschi 2004) of Engdahl et al.'s (1998) database (figure 1b), or earlier P- and S-velocity models (Becker \& Boschi 2002, and their additional on-line material).

This means that the vertical coherence of Montelli et al.'s (2004a) model is a global feature, not limited to plumes/hot-spots. It remains high even across the $660 \mathrm{~km}$ discontinuity, where a change in the pattern of heterogeneity has been observed in a number of models (Becker \& Boschi 2002), and can be explained in terms of independent geophysical considerations (e.g., Puster \& Jordan 1997). 
It is possible that the anomalously high radial coherence in figure 1a be an artifact, and some questions arise: is this feature equally required by the observations of Engdahl et al. (1998) and those of Bolton \& Masters (2001), both inverted by Montelli et al. (2004a)? Independent models, based on Engdahl et al.'s (1998) data, are remarkably vertically uncoherent in and below the transition zone (Becker \& Boschi 2002); is the regularization/parameterization strategy (Nolet \& Montelli 2005) employed by the Princeton authors appropriate? In practice, the radial smoothness of a tomographic model is often governed by an independent vertical roughness operator. In Montelli et al.'s (2004a) approach, only one damping parameter controls both radial and horizontal smoothness (eq. (12) of Montelli et al. 2004b); this narrows the portion of solution space explored, and might lead to excluding acceptable solutions of lower radial smoothness. In the absence of a-priori information on the shape of velocity anomalies, the isotropic smoothing criterion is legitimate; on the other hand, teleseismic $\mathrm{P}$ waves systematically sample the Earth's mantle horizontally over longer distances than vertically: it might be useful to check the response of the solution to other regularization schemes.

\section{Does Born Theory Enhance Resolution? A Surface Wave Experiment}

In view of figure 1, and of the works of de Hoop \& van der Hilst (2005b) and van der Hilst \& de Hoop (2005), we decided to conduct an independent evaluation of the improvement in resolution achieved by Born-theory vs. ray-theory tomography, addressing the issue of comparing Born- vs. ray-theoretical tomographic images. We limit ourselves to surface wave phase velocity tomography (a smaller, two-dimensional problem), but our approach can be extended to the body-wave, 3-D case. 
As discussed at some length by Boschi (2006, section 4), "the introduction of Borntheoretical kernels tends to lower the mean value of diagonal entries [of the tomographic "A $A$ A" matrix], but simultaneously broaden their range, with higher maxima and lower minima. This is the expected effect of a redistribution of tomographic resolution, owing to the higher sensitivity [in the Born-theory case] in the immediate vicinity of sources and stations, where [the finite-frequency Fréchet kernel] is singular". In other words: for a comparison between the results of ray- and Born-theory tomography to be meaningful, one should carry out the two inversions in exactly the same way: same data coverage, same parameterization, same regularization, same inversion algorithm. Unfortunately, inherent differences between the two theories make it impossible to identify equivalent regularization schemes for the two cases. This ultimately prevents a rigorous comparison. We find that this difficulty has not been given, yet, the attention it deserves.

While direct, visual comparisons between tomographic maps are so hindered, a rigorous comparison between trade-off-, or L-curves (Hansen 1992) resulting from sets of differently regularized ray- and Born-theory inversions is possible. We conduct independent ray- and Born-theory inversions of the Harvard surface wave dispersion database (Ekström et al. 1997), at each observed period (35 s to $150 \mathrm{~s}$ ), to find 2-D phase velocity maps with 9 different 2-D parameterizations $\left(15^{\circ}, 10^{\circ}, 7.5^{\circ}, 6^{\circ}, 5^{\circ}, 3.75^{\circ}, 3^{\circ}, 2.5^{\circ}\right.$ and $2^{\circ}$ grids $)$. Borntheory kernels are defined as in Spetzler et al. (2002). At each parameterization level, we perform numerous inversions varying the value of the roughness-damping parameter (no other regularization constraint is imposed), so that a wide range of solutions, from 
roughness $\sim 0$ to the highest possible roughness before the inversion algorithm ceases to converge, are found.

All the plots in figure 2 are equivalent to the L-curves presented by Montelli et al. (2004b, figure 7), in that, at any given value of misfit, the Born-theory-based map is more complicated than the ray-theory one; or, at any given level of model complexity, the ray-theory solution achieves a better datafit than the Born-theory one. If we believe Born theory (in Spetzler et al.'s (2002) 2-D formulation, and applied to Ekström et al.'s (1997) measurements) to be better than ray theory, we must infer from figure 2 that Born-theory tomography allows to constrain structures of higher complexity than ray theory; equivalently, that Born theory should allow to constrain a larger number of free parameters than ray theory. We verify this in a rigorous fashion. While $\chi^{2}$ or variance reduction vary monotonically as functions of the number of free parameters, the Akaike information criterion (Akaike 1974; for applications to Earth sciences see, e.g., Oda \& Shibuya 1996; Zollo et al. 2002; Dal Forno et al. 2005) provides a measure of model likelihood that is maximum when the optimal number of free parameters is inverted for, and decreases in regimes of both under- and over-fitting. The number of free parameters that a database, in a given theoretical formulation, can reasonably constrain, can then be identified by finding the maximum of a function dubbed AICC (corrected Akaike information criterion: Hurvich \& Tsai 1989).

After normalizing model roughness to its highest found value for each formulation and at each parameterization level, we calculate the curvature (figure 3) of the L-curves found above, and pick as our favored solutions (preferred damping) those corresponding to max- 
imum curvature. We next find the associated resolution matrix R (e.g., Menke 1989). Because of the sparsity of the tomographic matrix resulting from the pixel parameterization, R is best calculated via a set of independent LSQR (Paige \& Saunders 1982) inversions (as many as there are pixels in our grid) performed in parallel on the nodes of (for example) a Beowulf cluster (e.g. Boschi et al. 2006). The trace of R is an estimate of the number of degrees of freedom, or effective number of free parameters in the solution (Tarantola 2005, section 3.3.2); we use it to calculate AICC and illustrate the results in figure 4.

At all considered surface wave modes, AICC grows quickly for coarse parameterizations, with a corner corresponding to the $5^{\circ}$ grid, including 1656 equal-area pixels or between 250 and 400 effective degrees of freedom. AICC remains approximately constant at parameterization levels of $3^{\circ}$ or higher. While AICC curves resulting from Born and ray theory have a similar character, the Born-theory ones reach their plateau at slightly higher values of $\operatorname{tr}(\mathrm{R})$. This result, albeit only marginal in this case, indicates that Born-theory tomography is capable of constraining a higher number of free parameters.

\section{Conclusions}

We have found independent Born-theoretical and ray-theoretical solutions to the surface-wave phase velocity $(2-\mathrm{D})$ inverse problem on a set of different parameterizations $\left(15^{\circ}\right.$ - to $2^{\circ}$-pixel grids), and used the corrected Akaike information criterion (Hurvich \& Tsai 1989) to identify the effective number of free parameters constrained by the two formulations. Particularly at longer periods, the highest-likelihood Born-theory solutions are achieved with higher numbers of degrees of freedom (independent solution coefficients) 
than their ray-theory counterparts (figure 4). This explains the controversial result of our L-curve analysis (figure 2), with Born-theory surface-wave tomography favoring solutions of higher complexity than ray theory.

The picture will change when surface-wave phase anomaly observations are inverted to find 3-D maps of shear velocity in the upper mantle. The corresponding sensitivity kernels are less oscillatory/have less prominent sidebands than ours, and are adequately described by a relatively coarse parameterization. The inverse problem accordingly becomes more stable, and Zhou et al.'s (2005) Born-theoretical upper mantle models achieve a better datafit than ray-theory ones, at the same level of model complexity (Zhou et al. 2005, figure 4).

P-wave, Born-theory based kernels (Dahlen et al. 2000) employed by Montelli et al. (2004a) have almost negligible sidebands, so that the parameterization issue should be less important. Nevertheless, the L-curves found by Montelli et al. (2004b, figure 7) are equivalent to those of figure 2 here, with the ray-theory solution achieving a better datafit than the Born-theory one, at any given level of model complexity.

We suggest that applying the information-theory analysis of figure 4 to the inverse problem of Montelli et al. (2004a) might serve to better evaluate the significance of differences between ray- and Born-theory P-velocity tomography.

Acknowledgments. We thank Jean-Paul Ampuero, Tony Dahlen, Giulio Dal Forno, Göran Ekström and Guust Nolet for sharing their ideas; Raffaella Montelli for making her model available to us. LB wishes to thank Domenico Giardini for his constant support and encouragement. Our original manuscript has been improved thanks to the comments of 
Aldo Zollo and two anonymous reviewers. This work has benefited from LB's participation in the EU Research Training Network SPICE. TWB was partly supported by NSF grants EAR-0330717 and 0409373.

\section{References}

Akaike, H., 1974. A new look at the statistical model identification, IEEE Trans. Autom. Contr., 19, 716-723.

Antolik, M., Y. J. Gu, G. Ekström, and A. M. Dziewonski, 2003. J362D28: A new joint model of compressional and shear wave velocity in the Earth's mantle, Geophys. J. Int., 153, 443-466.

Becker, T. W., and L. Boschi, 2002. A comparison of tomographic and geodynamic mantle models, Geochem. Geophys. Geosyst., 3, DOI:10.1029/2001GC000168.

Bolton, H., and G. Masters, 2001. Travel times of P and S from the global digital seismic networks: Implications for the relative variation of $\mathrm{P}$ and $\mathrm{S}$ velocity in the mantle, $J$. Geophys. Res., 106, 13527-13540.

Boschi, L., 2006. Global multi-resolution models of surface wave propagation: the effects of scattering, Geophys. J. Int., submitted.

Boschi, L., and A. M. Dziewonski, 1999. "High" and "low" resolution images of the Earth's mantle: Implications of different approaches to tomographic modeling, J. Geophys. Res., 104, 25,567-25,594.

Boschi, L., G. Soldati, and A. Piersanti, 2006. Global seismic tomography and modern parallel computers, Ann. Geophys., submitted. 
Dahlen, F. A., S. H. Hung, and G. Nolet, 2000. Fréchet kernels for finite-frequency traveltimes-I. Theory, Geophys. J. Int., 141, 151-174.

Dahlen, F. A., and G. Nolet, 2005. Comment on "on sensitivity kernels for wave-equation transmission tomography" by M. V. de Hoop and R. D. van der Hilst, Geophys. J. Int., in press.

Dal Forno, G., P. Gasperini, and E. Boschi, 2005. Linear or nonlinear rheology in the mantle: a 3D finite-element approach to postglacial rebound modeling, J. Geodyn., 39, $183-195$.

de Hoop, M. V., and R. D. van der Hilst, 2005a. On sensitivity kernels for wave-equation transmission tomography, Geophys. J. Int., 160, 621-633.

de Hoop, M. V., and R. D. van der Hilst, 2005b. Reply to comment by F. A. Dahlen and G. Nolet on: "on sensitivity kernels for wave-equation transmission tomography", Geophys. J. Int., 163, 952-955.

Dziewonski, A.M., and D. L. Anderson, 1981. Preliminary reference Earth model, Phys. Earth Planet. Inter., 25, 297-356.

Ekström, G., J. Tromp, and E.W.F. Larson, Measurements and global models of surface wave propagation, J. Geophys. Res., 102, 8137-8157, 1997.

Engdahl, E. R., R. D. van der Hilst, and R. P. Buland, 1998. Global teleseismic earthquake relocation with improved travel times and procedures for depth determination, Bull. Seismol. Soc. Am., 88, 722-743.

Favier, N., S. Chévrot, and D. Komatitsch, 2004. Near-field influence on shear wave splitting and traveltime sensitivity kernels, Geophys. J. Int., 156, 467-482. 
Friederich, W., 1999. Propagation of seismic shear and surface waves in a laterally heterogeneous mantle by multiple forward scattering, Geophys. J. Int., 136, 180-204.

Hansen, P. C., 1992. Analysis of discrete ill-posed problems by means of the L-curve, SIAM Rev., 34, 561-580.

Hung, S. H., F. A. Dahlen, and G. Nolet, 2000. Fréchet kernels for finite-frequency traveltimes-II. Examples, Geophys. J. Int., 141, 175-203.

Hurvich, C. M., and C.-L. Tsai, 1989. Regression and time series model selection in small samples, Biometrika, 76, 297-307.

Kárason, H., and R. D. van der Hilst, 2001. Tomographic imaging of the lowermost mantle with differential times of refracted and diffracted core phases (PKP, Pdiff), J. Geophys. Res., 106, 6569-6588.

Li, X.-D., and B. Romanowicz, 1996. Global mantle shear-velocity model developed using nonlinear asymptotic coupling theory, J. Geophys. Res., 101, 22,245-22,272.

Li, X.-D., and T. Tanimoto, 1993. Waveforms of long-period body waves in a slightly aspherical Earth model, Geophys. J. Int., 112, 92-102.

Menke, W., 1989. Geophysical Data Analysis: Discrete Inverse Theory, rev. ed., Academic, San Diego.

Montelli, R., G. Nolet, F. A. Dahlen, G. Masters, E. R. Engdahl, and S.-H. Hung, 2004a. Finite-frequency tomography reveals a variety of plumes in the mantle, Science, $\mathbf{3 0 3}$, $338-343$.

Montelli, R., G. Nolet, G. Masters, F. A. Dahlen, and S.-H. Hung, 2004b. Global P and PP traveltime tomography: rays versus waves, Geophys. J. Int., 158, 637-654, DOI: 
10.1111/j.1365-246X.2004.02346.x.

Nolet, G., and R. Montelli, 2005. Optimal parameterization of tomographic models, Geophys. J. Int., 161, 365-372, DOI: 10.1111/j.1365.246X.2005.02596.x.

Oda, H., and H. Shibuya, 1996. Deconvolution of long-core paleomagnetic data of Ocean Drilling Program by Akaike's Bayesian Information Criterion minimization, J. Geophys. Res., 101, 2815-2834.

Paige, C. C., and M. A. Saunders, 1982. LSQR: an algorithm for sparse linear equations and sparse least squares, ACM Trans. Math. Soft., 8, 43-71.

Puster, P., and T. H. Jordan, 1997. How stratified is mantle convection?, J. Geophys. Res., 102, 7625-7646.

Ritsema, J., H. J. van Heijst, and J. H. Woodhouse, 1999. Complex shear wave velocity structure imaged beneath Africa and Iceland, Science, 286, 1925-1928.

Sieminski, A. J.-J. Lévêque, and E. Debayle, 2004. Can finite-frequency effects be accounted for in ray theory surface wave tomography?, Geophys. Res. Lett., 31, DOI:10.1029/2004GL021402.

Snieder, R., and G. Nolet, 1987. Linearized scattering of surface waves on a spherical Earth, J. Geophys., 61, 55-63.

Soldati, G., and L. Boschi, 2004. Whole Earth tomographic models: a resolution analysis Eos Trans. AGU, 85, Fall Meet. Suppl., Abstract S13D-1092.

Spetzler, J., J. Trampert, and R. Snieder, 2001. Are we exceeding the limits of the great circle approximation in surface wave tomography?, Geophys. Res. Lett., 28, 2341-2344. 
Spetzler, J., J. Trampert, and R. Snieder, 2002. The effect of scattering in surface wave tomography, Geophys. J. Int., 149, 755-767.

Tarantola, A., 2005. Inverse Problem Theory and Model Parameter Estimation, SIAM, Philadelphia.

Trampert, J., and J. H. Woodhouse, 2001. Assessment of Global Phase Velocity Models, Geophys. J. Int., 144, 165-174.

van der Hilst, R. D., and M. V. de Hoop, 2005. Banana-doughnut kernels and mantle tomography, Geophys. J. Int., 163, 956-961.

Woodhouse, J. H., and T. P. Girnius, 1982. Surface waves and free oscillations in a regionalized earth model, Geophys. J. R. Astron. Soc., 68, 653-673.

Yoshizawa, K., and B. L. N. Kennett, 2005. Sensitivity kernels for finite-frequency surface waves, Geophys. J. Int., 162, 910-926, DOI:10.1111/j.1365-246X.2005.02707.x.

Zhao, L., T. H. Jordan, and C. H. Chapman, 2000. Three-dimensional Fréchet differential kernels for seismic delay times, Geophys. J. Int., 141, 558-576.

Zhou, Y. F. A. Dahlen, G. Nolet, and G. Laske, 2005. Finite-frequency effects in global surface-wave tomography, Geophys. J. Int., 163, 1087-1111, doi:10.1111/j.1365246X.2005.02780.x.

Zollo, A., L. D'Auria, R. De Matteis, A. Herrero, J. Virieux and P. Gasparini, 2002. Bayesian estimation of 2-D P-velocity models from active seismic arrival time data: imaging of the shallow structure of Mt Vesuvius (Southern Italy), Geophys. J. Int., $151,566-582$. 


\section{Figures}
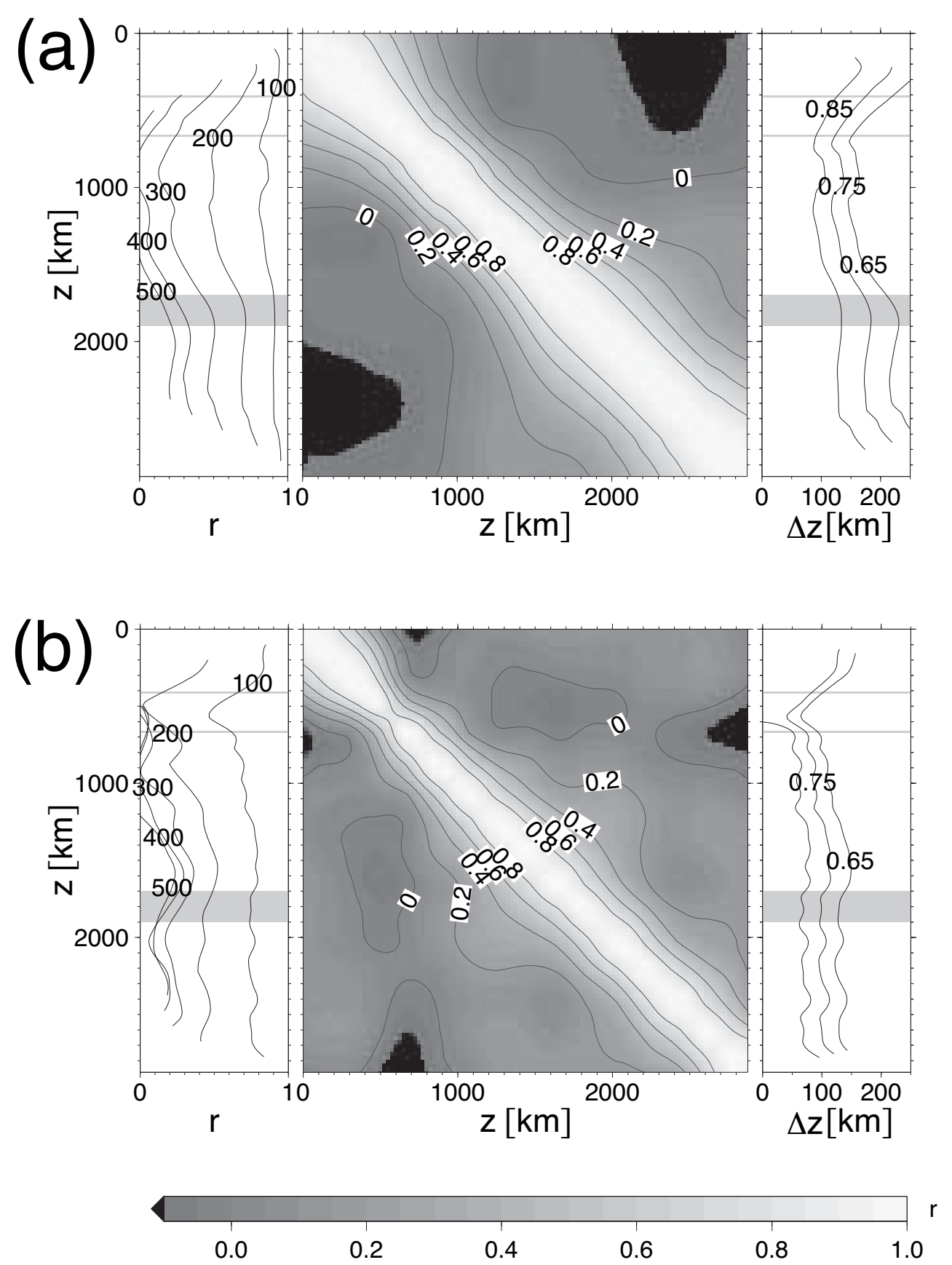

Figure 1. Radial correlation function $r$ for (a) an updated version of Montelli et al.'s (2004a) model, with improved crustal correction (R. Montelli, personal communication, 2005), and (b) updated version of model BDP98 (Boschi \& Dziewonski 1999; Soldati \& Boschi 2004) after relocation and crustal correction as described by Antolik et al. (2003). We show $r$ at fixed depths of 100-500 km (left plots), radial correlation matrix $r(z 1, z 2)$ with contours in 0.2 intervals (middle plots), and depth intervals $\Delta z$ for fixed $r=0.65, r=0.75$, and $r=0.85$ (right plots). In analogy with Becker \& Boschi (2002), the $410 \mathrm{~km}$ and $660 \mathrm{~km}$ discontinuities, and the 1700-1900 km depth range are indicated. 


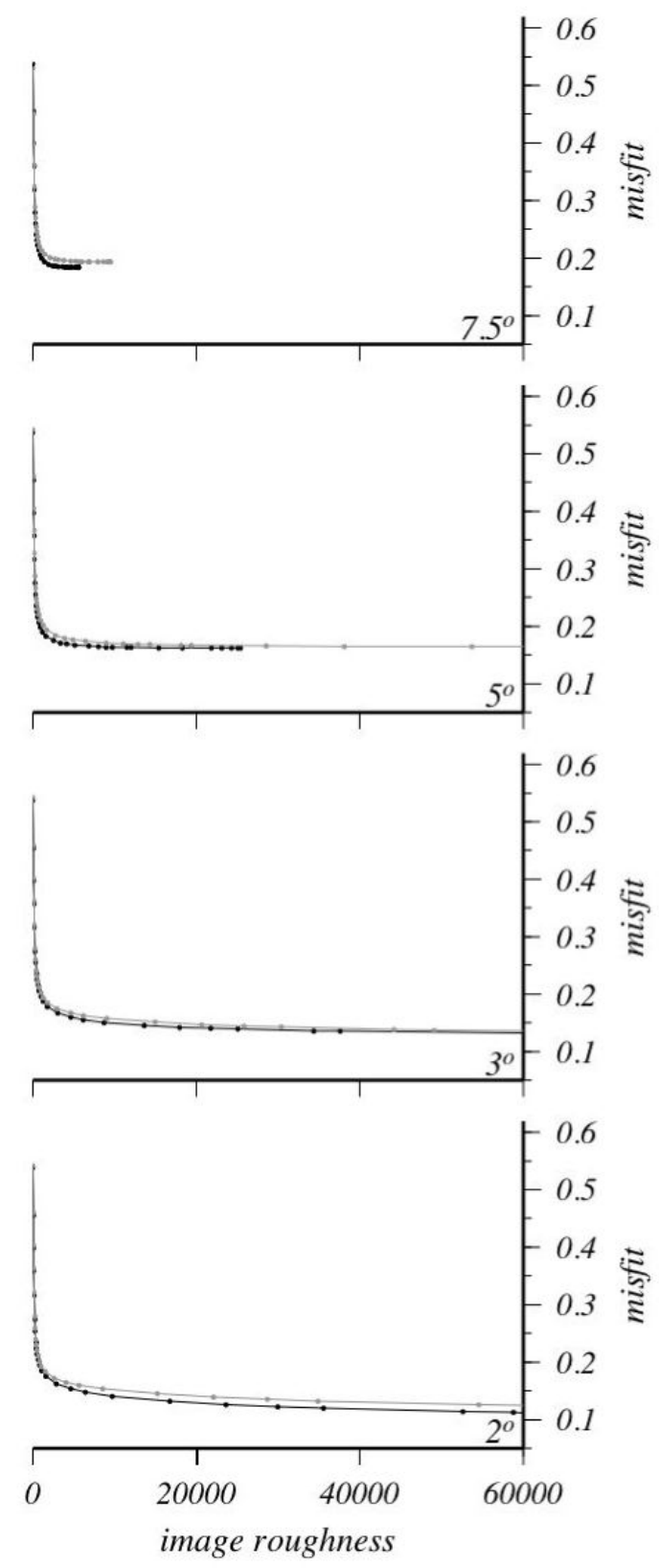

Figure 2. Example of L-curve analysis of surface-wave phase-anomaly inversions from the Harvard database, for 100s Love waves: inversions are performed covering a broad range of roughness damping parameter values (no other regularization applied), and, for each inversion, data misfit (1-variance reduction) is plotted vs. image roughness (global integral of the squared modulus of the gradient). Acceptable models lie near the L-curve's corner. L-curves are found for different parameterizations (longitudinal size of pixels in degrees is specified on each plot), both in the ray- (black dots/lines) and Born-theory (gray) cases. Born-theory L-curves are above ray-theory ones: ray-theory solutions fit the data better than Born-theory solutions of equal complexity. 


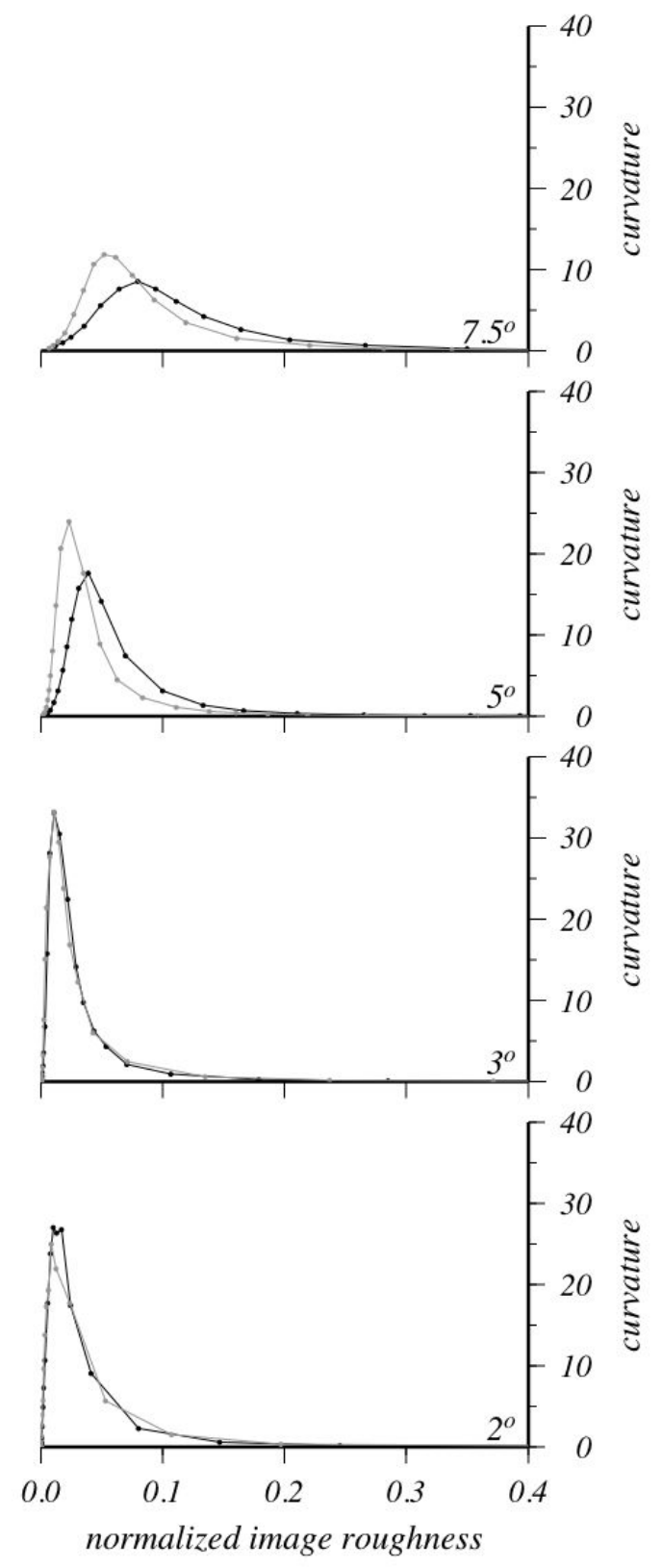

Figure 3. Curvature of the L-curves shown in figure 2, with gray denoting Born-, and black ray-theory results. Before calculating the curvature, we normalize each L-curve to the value of model roughness associated with its rightmost point (Born- and ray- theory L-curves are normalized independently). Our favored solutions, to be employed in the subsequent Akaike analysis, are those corresponding to maximum curvature. 

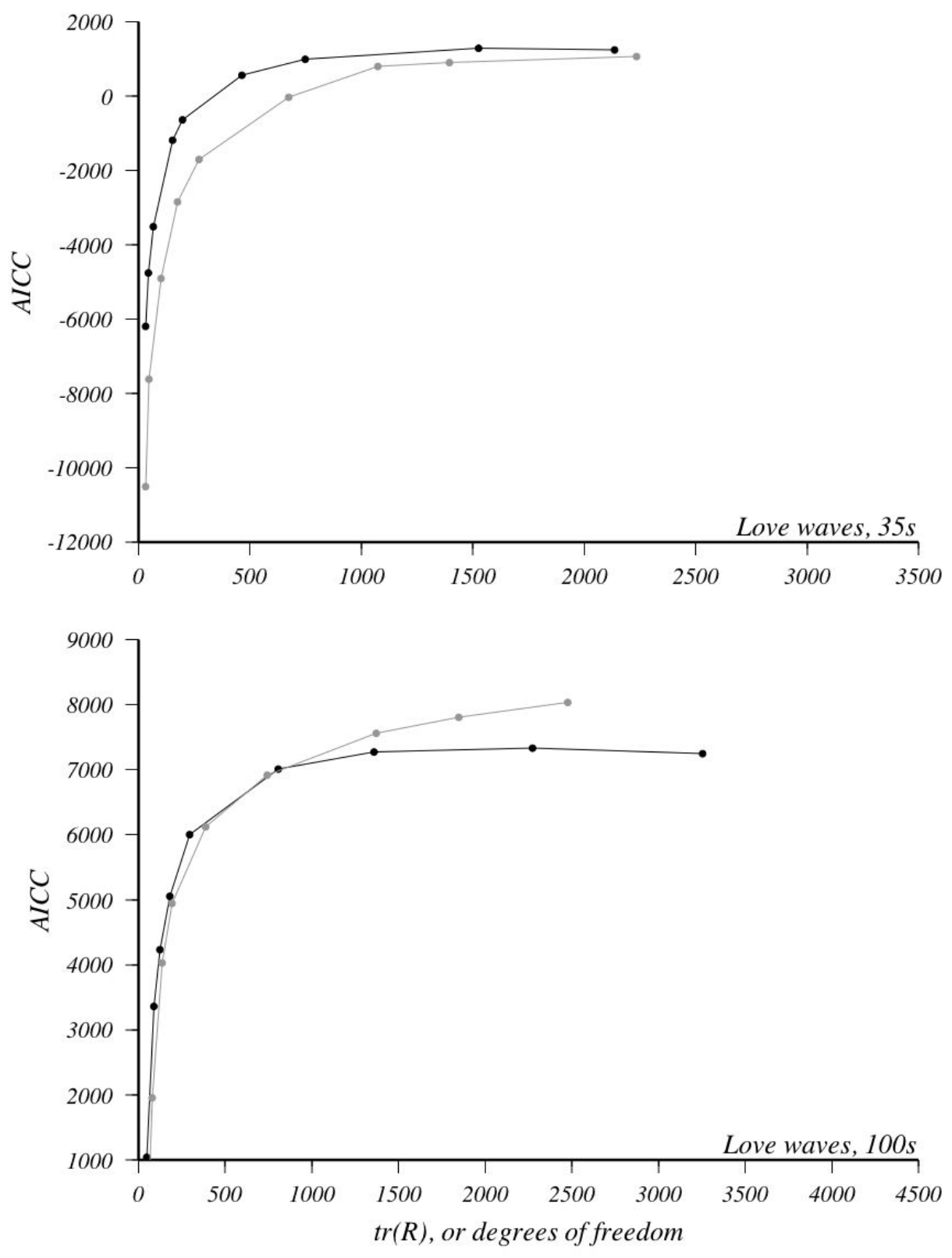

Figure 4. Examples of the information content (Hurvich \& Tsai 1989) of selected ray- (black dots/lines) and Born-theory (gray) solutions, for Love waves at $35 \mathrm{~s}$ (top panel) and $100 \mathrm{~s}$ (bottom) periods. 